\title{
A GENERALIZATION OF A COMBINATORIAL THEOREM OF SPARRE ANDERSEN ABOUT SUMS OF RANDOM VARIABLES
}

\section{ACHI BRANDT}

\section{Introduction.}

We consider a finite or infinite sequence $X_{1}, X_{2}, \ldots$ of real valued random variables. The random variables $X_{1}, \ldots, X_{n}$ are said to be symmetrically dependent if

$$
\operatorname{Pr}\left[\bigcap_{i=1}^{n}\left\{X_{i} \leqq x_{i}\right\}\right]=\operatorname{Pr}\left[X_{i} \leqq x_{i}, i=1,2, \ldots, n\right]
$$

is a symmetric function of $x_{1}, \ldots, x_{n}$. If an event $C$ is invariant under permutation of the variables $x_{1}, \ldots, x_{n}$ we say that the event is symmetric with respect to $X_{1}, \ldots, X_{n}$. For $n=1,2,3, \ldots$, we introduce the following random variables:

$$
\begin{aligned}
& S_{n}=X_{1}+\ldots+X_{n}, \quad S_{0}=0 . \\
& R_{n}=\max \left[S_{0}, S_{1}, \ldots, S_{n}\right], \quad R_{0}=0 .
\end{aligned}
$$

$N_{n}(\gamma)=$ the number of sums $S_{1}, \ldots, S_{n}$ which are greater than $\gamma$.

$L_{n}(\gamma)$; for $\gamma \geqq 0$ we define $L_{n}(\gamma)$ as the first index $k, 0 \leqq k \leqq n$, for which $S_{k} \geqq R_{n}-\gamma$, and for $\gamma<0, L_{n}(\gamma)$ is defined to be the last index $k, 0 \leqq k \leqq n$, for which $S_{k}>R_{n}+\gamma$.

The main result of this note is

TheORem 1.1. If $X_{1}, \ldots, X_{n}$ are symmetrically dependent random variables, and $C$ is an event which is symmetric with respect to $X_{1}, \ldots, X_{n}$, then

$$
\text { (1.1) } \operatorname{Pr}\left[N_{n}(\gamma)=k, C\right]=\operatorname{Pr}\left[L_{n}(\gamma)=k, C\right], k=0,1, \ldots, n,-\infty<\gamma<\infty \text {. }
$$

The case $\gamma=0$ is a known theorem of E. Sparre Andersen, who proved it by induction (see [4, Theorem 1]). Other proofs for $\gamma=0$ have been given by F. Spitzer [5] and W. Feller [2]. Our method is entirely combinatorial, and related to the methods in [5] and [2].

The combinatorial tool required to prove Theorem 1.1 is developed in Section 2. Since this tool (ordered mixture) may have some interest 
in itself, we give the theorems in Sec. 2 in more general form than necessary for our purposes. In Section 3 we prove Theorem 1.1. In Section 4 certain corollaries, dealing with the order statistics of the sums $S_{1}, S_{2}, \ldots$, are obtained. These are related to the works of Wendel [6] and Pollaczec [3].

The author is indebted to $\mathrm{H}$. Kesten for valuable suggestions.

\section{Ordered Mixture.}

Definition. Let $A$ be a finite set of finite sequences,

$$
A=\left\{\left(x_{i, 1}, x_{i, 2}, \ldots, x_{i, n_{i}}\right) ; i=1,2, \ldots, m\right\} .
$$

A sequence $y=\left(y_{1}, \ldots, y_{n}\right)$ is called an ordered mixture (o.m.) of $A$ if there is a 1-1 correspondence

$$
(i, j) \leftrightarrow v=v(i, j),
$$

$j=1, \ldots, n_{i}, i=1, \ldots, m, v=1, \ldots, n$ so that $x_{i, j}=y_{v(i, j)}$ and $v(i, j+1)>$ $v(i, j)$ for $j=1,2, \ldots, n_{i}-1$; that is, the members of $y$ are exactly all the $x_{i, j}$, and the order of the members of each sequence of $A$ is preserved in $y$.

For example, the sequence $(1,4,5,2,7,6,8,3)$ is an o.m. of the set $\{(1,2,3),(4,5,6),(7,8)\}$.

Theorem 2.1. Let $\left(E_{1}, \ldots, E_{m}\right)$ be a disjoint sequence of sets of real numbers, and suppose $A$ is any sequence of real sequences

$$
A=\left(\xi_{1}, \xi_{2}, \ldots, \xi_{m}\right), \quad \xi_{i}=\left(x_{i_{1}}, x_{i_{2}}, \ldots, x_{i_{n_{i}}}\right), 1 \leqq i \leqq m .
$$

Then there is at most one o.m. of $A$, say $y=\left(y_{1}, \ldots, y_{n}\right)$, for which

$$
\sum_{m=1}^{v(i, j)} y_{m} \in E_{i}, \quad v(i, j)=1,2, \ldots, n
$$

Proof. The proof is easily accomplished by induction with respect to $\sum_{1}^{m} n_{i}$ ( $m$ being fixed throughout the induction; $n_{i}$ may be zero). The case $\sum n_{i}=1$ is trivial. We assume now that the theorem holds for $\sum n_{i}=n-1$ and shall prove the case $\sum n_{i}=n$. Put

$$
\sum_{i=1}^{m} \sum_{j=1}^{n_{i}} x_{i, j}=S
$$

If an o.m. $\left(y_{1}, \ldots, y_{n}\right)$ with the above property does exist, then $\sum_{\mu=1}^{m} y_{\mu}=S$, which implies $S \in E_{k}$ and $y_{n} \in \xi_{k}$ for some $k$ with $1 \leqq k \leqq m$. Thus there are two possibilities: Either $S \notin \bigcup_{1}^{m} E_{i}$, in which case no o.m. exists at all; or $S \in E_{k}$ in which case we must have $y_{n}=x_{k, n_{k}}$, and then by the 
induction assumption there is at most one admissible arrangement of $y_{1}, \ldots, y_{n-1}$. This completes the proof.

A similar theorem with a similar proof is clearly valid for any group instead of the real numbers.

Definition. The ordered mixture $\left(y_{1}, \ldots, y_{n}\right)$ in Theorem 2.1 is called the ordered mixture of $\left(\xi_{1}, \ldots, \xi_{m}\right)$ with respect to $\left(E_{1}, \ldots, E_{m}\right)$. Or, when abbreviated: $\left(y_{1}, \ldots, y_{n}\right)$ is the o.m. $\left(E_{1}, \ldots, E_{m}\right)$ of $\left(\xi_{1}, \ldots, \xi_{m}\right)$.

Theorem 2.2. Let $\left(E_{1}, \ldots, E_{m}\right)$ be a disjoint sequence of sets of real numbers, and let $X_{1}, \ldots, X_{n}$ be symmetrically dependent random variables. Furthermore, let $n_{1}, \ldots, n_{m}$ be non-negative integers, with $n_{1}+\ldots+n_{k}=N_{k}$, $N_{m}=n, N_{0}=0$. Then, for the partial sums $S_{1}, \ldots, S_{n}$ and the sequences

we have

$$
\xi_{k}=\left(X_{N_{k-1}+1}, X_{N_{k-1}+2}, \ldots, X_{N_{k}}\right), \quad k=1,2, \ldots, m,
$$

$$
\begin{aligned}
\operatorname{Pr}\left[\bigcap_{i=1}^{m}\left\{\text { exactly } n_{i} \text { partial sums are in } E_{i}\right\}\right] \\
\quad=\operatorname{Pr}\left[\left(\xi_{1}, \ldots, \xi_{m}\right) \text { has an o.m. }\left(E_{1}, \ldots, E_{m}\right)\right] .
\end{aligned}
$$

Proof. Let $\Sigma^{*}$ denote summation extending over all the $n$-tuples $\left\{j_{i, k} \mid 1 \leqq k \leqq n_{i}, 1 \leqq i \leqq m\right\}$ for which $1 \leqq j_{i, 1}<\ldots<j_{i, n_{i}} \leqq n, i=1,2, \ldots, m$, and whose members $j_{i, k}$ are all distinct. Then

$$
\begin{aligned}
\operatorname{Pr}\left[\bigcap_{i=1}^{m}\left\{\text { exactly } n_{i} \text { partial sums are in } E_{i}\right\}\right] \\
=\Sigma^{*} \operatorname{Pr}\left[\bigcap_{i=1}^{m} \bigcap_{k=1}^{n_{i}}\left\{S_{j_{i, k}} \in E_{i}\right\}\right] \\
=\sum^{*} \operatorname{Pr}\left[X_{1}, \ldots, X_{n} \text { is the o.m. }\left(E_{1}, \ldots, E_{m}\right)\right. \text { of } \\
\quad\left(\left(X_{j_{1,1}}, X_{j_{1,2}}, \ldots, X_{j_{1, n_{1}}}\right),\left(X_{j_{2,1}}, X_{j_{2,2}}, \ldots, X_{j_{2, n_{2}}}\right), \ldots\right. \\
\left.\left.\ldots,\left(X_{j_{m, 1}}, X_{j_{m, 2}}, \ldots, X_{j_{m, n_{m}}}\right)\right)\right] \\
=\sum^{*} \operatorname{Pr}\left[\left(\xi_{1}, \ldots, \xi_{m}\right) \text { has an o.m. }\left(E_{1}, \ldots, E_{m}\right)\right. \text { in } \\
\left.\quad \text { which } x_{N_{k-1}+1} \text { appears in the } j_{k, 1} \text {-th place }\right] \\
=\operatorname{Pr}\left[\left(\xi_{1}, \ldots, \xi_{m}\right) \text { has an o.m. }\left(E_{1}, \ldots, E_{m}\right)\right] .
\end{aligned}
$$

In the third equality we made use of the symmetrical dependence of the random variables $X_{1}, \ldots, X_{n}$.

REMARK. All the above equalities remain valid if every event in brackets [...] is intersected with an event $C$ which is symmetric with respect to $X_{1}, \ldots, X_{n}$. 
In the following theorem $(\gamma, \infty)$ and $(-\infty, \gamma]$ denote the real intervals $\gamma<x<\infty$ and $-\infty<x \leqq \gamma$, respectively.

Theorem 2.3. Let $x=\left(x_{1}, \ldots, x_{L}\right)$ and $y=\left(y_{1}, \ldots, y_{M}\right)$ be two sequences of real numbers. Then for every real number $\gamma,(x, y)$ has an o.m. $((\gamma, \infty)$, $(-\infty, \gamma])$ which begins with $x_{1}$, if and only if

where

$$
Q+P \leqq \gamma \quad \text { and } \quad P>\gamma
$$

$$
Q=\max _{1 \leqq m \leqq M} \sum_{1}^{m} y_{v}, \quad P=\min _{1 \leqq l \leqq L} \sum_{1}^{l} x_{v} .
$$

Proof. Suppose $(x, y)$ has an o.m. $((\gamma, \infty),(-\infty, \gamma])$ of the form

$$
\begin{gathered}
x_{1}, x_{2}, \ldots, x_{i_{1}} ; \quad y_{1}, y_{2}, \ldots, y_{j_{1}} ; \quad x_{i_{1}+1}, x_{i_{2}+2}, \ldots, x_{i_{2}} ; \\
y_{j_{1}+1}, y_{j_{1}+2}, \ldots, y_{j_{2}} ; \quad \ldots
\end{gathered}
$$

where $0<i_{1}<i_{2}<\ldots, 0=j_{0}<j_{1}<j_{2}<\ldots$ By the definition of o.m. we have

and, for $i_{\alpha}<k \leqq j_{\alpha+1}$,

$$
\sum_{1}^{i_{\alpha}} x_{\nu}+\sum_{1}^{j_{\alpha}} y_{\nu} \leqq \gamma
$$

$$
\sum_{1}^{k} x_{v}+\sum_{1}^{j_{\alpha}} y_{v}>\gamma
$$

which together imply, for $\alpha \geqq 1$,

$$
\sum_{i_{\alpha}+1}^{k} x_{\nu}>0
$$

Thus, if $\Sigma_{1}^{\lambda} x_{\nu}=P$, then necessarily $\lambda \leqq i_{1}$, and therefore $\Sigma_{1}^{\lambda} x_{\nu}$ is a partial sum of the o.m., which implies $\Sigma_{1}^{\lambda} x_{\nu}=P>\gamma$.

Let $Q=\Sigma_{1}^{\mu} y_{v}$. For some $\alpha, j_{\alpha-1}<\mu \leqq j_{\alpha}$. Then, by the requirements of o.m.,

$$
P+Q \leqq \sum_{1}^{i_{\alpha}} x_{\nu}+\sum_{1}^{\mu} y_{\nu} \leqq \gamma .
$$

To prove the "if" assertion of the theorem, we construct the o.m. $\left(z_{1}, z_{2}, \ldots, z_{M+L}\right)$ starting at the right end, by the following procedure: We put

according as

$$
z_{M+L}=x_{L} \quad \text { or } \quad z_{M+L}=y_{M}
$$

$$
\sum_{1}^{L} x_{v}+\sum_{1}^{M} y_{v} \quad \text { falls in }(\gamma, \infty) \text { or in }(-\infty, \gamma] \text {. }
$$

Assume we have already defined $z_{m+l+1}, z_{m+l+2}, \ldots, z_{M+L}$, using $x_{l+1}$, $x_{l+2}, \ldots, x_{L}$ and $y_{m+1}, y_{m+2}, \ldots, y_{M}$. We then choose $z_{m+l}=x_{l}$ if $\Sigma_{1}^{l} x_{v}+\sum_{1}^{m} y_{v}>\gamma$ and $z_{m+l}=y_{m}$ otherwise. The sequence $z_{1}, \ldots, z_{M+L}$ so 
built is clearly an o.m. of $(x, y)$, but we have to prove that we can indeed proceed with the above construction until we get $z_{1}=x_{1}$.

There are no difficulties to define $z_{m+l}$ as long as both $m>0$ and $l>0$. If $m=0$, the choice $z_{l}=x_{l}$ agrees with the requirements, since $\Sigma_{1}^{l} x_{\nu} \geqq P>\gamma$. Thus, to complete the proof, we only have to show that it is impossible to reach a point where $l=0, m>0$, i.e. the case $z_{k+1}=x_{1}$, $k \geqq 1$. Such a situation indeed leads to a contradiction: Let $P=\sum_{1}^{\lambda} x_{v}$. For some $r \geqq k \geqq 1$ we have $x_{\lambda}=z_{r+\lambda}$, which would imply $\sum_{1}^{\lambda} y_{v}+\sum_{1}^{\lambda} x_{v}>\gamma$, in contradiction to the assumption $Q+P \leqq \gamma$. This completes the proof of Theorem 2.3.

The proof of the following theorem is essentially the same as that of the last one.

Theorem 2.4. In the above notation, $(x, y)$ has an o.m. $((\gamma, \infty),(-\infty, \gamma])$ that begins with $y_{1}$, if and only if

$$
Q+P>\gamma, \quad Q \leqq \gamma .
$$

\section{Proof of Theorem 1.1.}

The events $\left[N_{n}(\gamma)=0\right]$ and $\left[L_{n}(\gamma)=0\right]$ are identical, while $\left[N_{n}(\gamma)=n\right]$ is the image of the event $\left[L_{n}(\gamma)=n\right]$ under the permutation $X_{i} \rightarrow X_{n-i+1}$, $i=1, \ldots, n$. Thus we may restrict ourselves to the case $0<k<n$.

LEMmA 3.1. If $X_{1}, \ldots, X_{n}$ are symmetrically dependent random variables with respect to which $C$ is a symmetric event, then, for $0<k<n$,

$$
\begin{aligned}
& \operatorname{Pr}\left[N_{n}(\gamma)=k, C\right] \\
& =\operatorname{Pr}\left[\max _{k+1 \leqq m \leqq n} \sum_{k+1}^{m} X_{v}+\min _{1 \leqq l \leqq k} \sum_{1}^{l} X_{v} \leqq \gamma, \min _{1 \leqq l \leqq k} \sum_{1}^{l} X_{v}>\gamma, C\right]+ \\
& \quad+\operatorname{Pr}\left[\max _{k+1 \leqq m \leqq n} \sum_{k+1}^{m} X_{v}+\min _{1 \leqq l \leqq k} \sum_{1}^{l} X_{v}>\gamma, \max _{k+1 \leqq m \leqq n} \sum_{k+1}^{m} X_{v} \leqq \gamma, C\right] .
\end{aligned}
$$

This lemma is a direct corollary of Theorems 2.2 (with the subsequent remark), 2.3 and 2.4 .

To prove Theorem 1.1, let us first consider the case $\gamma \geqq 0$. By the permutation $X_{i} \rightarrow X_{k-i+1}, i=1,2, \ldots, k$, we derive from Lemma 3.1 that

$$
\operatorname{Pr}\left[N_{n}(\gamma)=k, C\right]=\operatorname{Pr}[\{Q+P \leqq \gamma, P>\gamma, C\} \cup\{Q+P>\gamma, Q \leqq \gamma, C\}],
$$
where

$$
\begin{gathered}
Q=Q_{k}=\max _{k+1 \leqq m \leqq n} \sum_{k+1}^{m} X_{v}, \\
P=P_{k}=\min _{1 \leqq l \leqq k} \sum_{l}^{k} X_{v}=S_{k}-R_{k-1} .
\end{gathered}
$$


Therefore, and from the fact that $Q+P \leqq \gamma$ and $P>\gamma$ implies $Q<0$ :

$\operatorname{Pr}\left[N_{n}(\gamma)=k, C\right]=$

$$
\begin{aligned}
& =\operatorname{Pr}[(\{Q+P \leqq \gamma, P>\gamma\} \cup\{Q+P>\gamma, Q \leqq \gamma, Q<0\} \cup\{Q+P>\gamma, Q \leqq \gamma, Q \geqq 0\}) C] \\
& =\operatorname{Pr}[(\{Q+P \leqq \gamma, P>\gamma, Q<0\} \cup\{Q+P>\gamma, Q<0, P>\gamma\} \cup\{Q+P>\gamma, 0 \leqq Q \leqq \gamma\}) C] \\
& =\operatorname{Pr}[(\{P>\gamma, Q<0\} \cup\{P+Q>\gamma, 0 \leqq Q \leqq \gamma\}) C] .
\end{aligned}
$$

Consequently, when $\gamma \geqq 0$, the theorem results from the following two trivial lemmas:

Lemma 3.2. Let $\gamma \geqq 0,0<k<n$. Then $Q_{k}<0$ and $P_{k}>\gamma$ if and only if $L_{n}(\gamma)=k$ and $S_{k}$ is the last maximum of the sequence $S_{1}, S_{2}, \ldots, S_{n}$.

Lemma 3.3. Let $\gamma \geqq 0,0<k<n$. Then $Q_{k}+P_{k}>\gamma$ and $0 \leqq Q_{k} \leqq \gamma$ if and only if $L_{n}(\gamma)=k$ and $S_{k}$ is not the last maximum of $S_{1}, S_{2}, \ldots, S_{n}$.

Note that $Q_{k}+P_{k}=\max _{k+1 \leqq i \leqq n} S_{i}-\max _{0 \leqq i \leqq k-1} S_{i}$.

Similarly, for $\gamma<0$ we have

$\operatorname{Pr}\left[N_{n}(\gamma)=k, C\right]$

$=\operatorname{Pr}[\{Q+P \leqq \gamma, P>\gamma, C\} \cup\{Q+P>\gamma, Q \leqq \gamma, C\}]$

$=\operatorname{Pr}[(\{Q+P \leqq \gamma, \gamma<P<0\} \cup\{Q+P \leqq \gamma, P>\gamma, P \geqq 0\} \cup\{Q+P>\gamma, Q \leqq \gamma\}) C]$

$=\operatorname{Pr}[(\{Q+P \leqq \gamma, \gamma<P<0\} \cup\{Q+P \leqq \gamma, P \geqq 0, Q \leqq \gamma\} \cup\{Q+P>\gamma, Q \leqq \gamma, P \geqq 0\}) C]$

$=\operatorname{Pr}[(\{Q+P \leqq \gamma, \gamma<P<0\} \cup\{P \geqq 0, Q \leqq \gamma\}) C]$,

and the corresponding lemmas:

Lemma 3.4. Let $\gamma<0,0<k<n$. Then $P_{k} \geqq 0$ and $Q_{k} \leqq \gamma$ if and only if $L_{n}(\gamma)=k$ and $S_{k}=R_{n}$.

Lemma 3.5. Let $\gamma<0,0<k<n$. Then $P_{k}+Q_{k} \leqq \gamma$ and $\gamma<P_{k}<0$ if and only if $L_{n}(\gamma)=k$ and $S_{k}<R_{n}$.

This completes the proof of Theorem 1.1 for all $\gamma$. Obviously, a similar theorem holds for $N_{n}^{*}(\gamma)=$ the number of sums $S_{1}, \ldots, S_{n}$ which are greater than or equal to $\gamma$.

\section{Corollaries.}

We introduce the notation: $S_{k}{ }^{+}=\max \left[S_{k}, 0\right]$. The order statistics of $S_{1}+, \ldots, S_{n}{ }^{+}$are designated by $R_{n, 1} \geqq R_{n, 2} \geqq R_{n, 3} \geqq \ldots \geqq R_{n, n}$.

Theorem 4.1. If $X_{1}, \ldots, X_{n}$ are symmetrically dependent random variables with respect to which $C$ is a symmetric event, then

$$
\operatorname{Pr}\left[R_{n, m+1} \leqq \gamma, C\right]=\operatorname{Pr}\left[R_{n}-R_{m} \leqq \gamma, C\right], \quad 0 \leqq m \leqq n-1,-\infty<\gamma<\infty .
$$


Proof. For $\gamma<0$ the two sides of the equality vanish. For $\gamma \geqq 0$ we just have to sum over $k=0,1,2, \ldots, m$ in (1.1).

The following theorem, concerning the characteristic functions of $\left(R_{n, k}, S_{n}\right)$ was first proved in [3] by function theoretic methods. In [6] an algebraic proof appeared which is closely connected with a recent proof in [1].

Theorem 4.2. (Pollaczec and Wendel.) Let $X_{1}, X_{2}, \ldots$ be an infinite sequence of independent and identically distributed random variables. Let $\Phi(\sigma)=E\left(\exp i \sigma X_{k}\right)$

$$
\begin{aligned}
\psi_{n} & =\psi_{n}(\varrho, \sigma)=E\left(\exp i\left[\varrho S_{n}++\sigma S_{n}\right]\right), \\
\zeta_{n, k} & =\zeta_{n, k}(\varrho, \sigma)=E\left(\exp i\left[\varrho R_{n, k}+\sigma S_{n}\right]\right) .
\end{aligned}
$$

Then, the following identity holds, provided $|\omega|,|z|<1$ :

$$
\sum_{n=1}^{\infty} \omega^{n} \sum_{k=1}^{n} z^{k-1} \zeta_{n, k}=\frac{1}{(1-z)(1-\omega z \Phi(\sigma))}\left[\exp \left\{\sum_{n=1}^{\infty} \frac{\omega^{n}}{n}\left(1-z^{n}\right) \psi_{n}\right\}-1\right] .
$$

It is not too difficult to prove this theorem from Theorem 1.1 (for $C$ we take the event $S_{n} \leqq t$ ) and a theorem of Spitzer. Spitzer's theorem is simply the case $z=0$ in Theorem 4.2 , but he has proved it independently (in [5]), using pure combinatorial considerations. Thus we obtain a purely combinatorial proof of Theorem 4.2. Conversely, combinatorial results may be deduced from Theorem 4.2 (see [5] and [7]) as well as from Spitzer's theorem (see [5]).

\section{REFERENCES}

1. G. Baxter, An analytic approach to finite fluctuation problems in probability, Applied Math. and Stat. Laboratories, Stanford University, California, July 29, 1960.

2. W. Feller, On combinatorial methods in fluctuation theory, The Harald Cramér Volume, Ed. Ulf Grenander, New York, 1959, 75-91.

3. F. Pollaczec, Fonctions caractéristiques de certaines repartitions définies au moyen de la notion d'ordre. Application a la théorie des attentes, C. R. Acad. Sci. Paris 234 (1952), 2334-2336.

4. E. Sparre Andersen, On sums of symmetrically dependent random variables, Skand. Aktuarietidskr. 36 (1953), 123-138.

5. F. Spitzer, A combinatorial lemma and its application to probability theory, Trans. Amer. Math. Soc. 82 (1956), 323-339.

6. J. G. Wendel, Order statistics of partial sums, Ann. Math. Stat. 31 (1960), 1034-1044.

7. J. G. Wendel, Spitzer's formula: a short proof, Proc. Amer. Math. Soc. 9 (1958), 905908. 